\begin{tabular}{|c|c|}
\hline Title & $\begin{array}{l}\text { Efficiency and limitation of periodic sample multiplication to reduce computational load in Monte Carlo simulations of } \\
\text { electron swarms in gas under attachment-dominated conditions }\end{array}$ \\
\hline Author(s) & Sugawara, Hirotake \\
\hline Citation & $\begin{array}{l}\text { Japanese Journal of A pplied Physics (JJA P), 57(3), } 038001 \\
\text { https://doi.org/10.7567/JJA P.57.038001 }\end{array}$ \\
\hline Issue Date & $2018-03$ \\
\hline Doc URL & http:/hdl.handle.net/2115/2415 \\
\hline Rights & @2018 The Japan Society of A pplied Physics \\
\hline Type & article (author version) \\
\hline File Information & Sugawara 2018-JJA P-57(3)-038001(3pp)-HU SCA P.pdf \\
\hline
\end{tabular}

Instructions for use 


\title{
Efficiency and limitation of periodic sample multiplication to reduce computational load in Monte Carlo simulations of electron swarms in gas under attachment-dominated conditions ${ }^{* \dagger}$
}

\author{
Hirotake Sugawara \\ Graduate School of Information Science and Technology, Hokkaido University, Sapporo 060-0814, Japan
}

In Monte Carlo simulations of electron swarms, sample electrons were copied periodically so that a sufficient number of samples are obtained in equilibrium after relaxation even under a severe attachment-dominated condition where most electrons vanish during the relaxation. The final sampling results were equivalent to those sampled by a conventional method, and the computational time conventionally wasted for the tracking of vanishing electrons was reduced drastically. The time saved can be utilized for tracking more samples to reduce statistical fluctuation. The efficiency of this technique and its limitation are discussed quantitatively together with details on its implementation.

Monte Carlo simulation (MCS) of electron swarms is a typical method to derive fundamental properties of discharge plasmas such as electron velocity distribution function (EVDF) and electron transport coefficients. Its advantage is that electron behavior can easily be taken into account; e.g., treatments for anisotropic scattering and gyration in magnetic fields would be complicated if considered in Boltzmann equation analyses. Instead, to reduce statistical fluctuation, a considerable computational load is required to track a sufficient number of sample electrons for a sufficiently long relaxation time to reach equilibrium. Empirically, we would need at least $10^{5}-10^{6}$ samples to obtain a fairly smooth EVDF. Mean electron energy and average velocity, for which the electron position is not considered, would be available stably from these populations. However, other electron transport coefficients require more samples; e.g., diffusion coefficient derived from the second-order spatial moment is sensitive to statistical fluctuation, and collision frequencies of rare reactions are essentially stochastic. $10^{7}-10^{8}$ electrons were sampled in MCSs in which a high precision was required. ${ }^{1-3}$

Attachment-dominated conditions are severe for MCS because the number of electrons decreases during the relaxation. ${ }^{4}$ The electron attachment in electronegative gases likely occurs for low-energy electrons. The number of electrons tends to decrease at low $E / N$ ( $E$, electric field; $N$, gas molecule number density) and/or high $B / N(B$, magnetic field) values. In addition, the relaxation is slow under such conditions. To overcome this difficulty, periodic sample multiplication (PSM) is investigated in this study. Similar treatments have often been adopted in MCSs as reviewed in Ref. 4, and related scaling techniques are also adopted in particle-in-cell MCSs. However, some important details are still left as unwritten knowledge. Because PSM is a beneficial but empirical technique, it is meaningful

\footnotetext{
*Published source: Japanese Journal of Applied Physics 57(3) 038001 (3 pages) (2018)

${ }^{\dagger}$ DOI: 10.7567/JJAP.57.038001

${ }^{\ddagger}$ E-mail: sugawara@ist.hokudai.ac.jp
} 


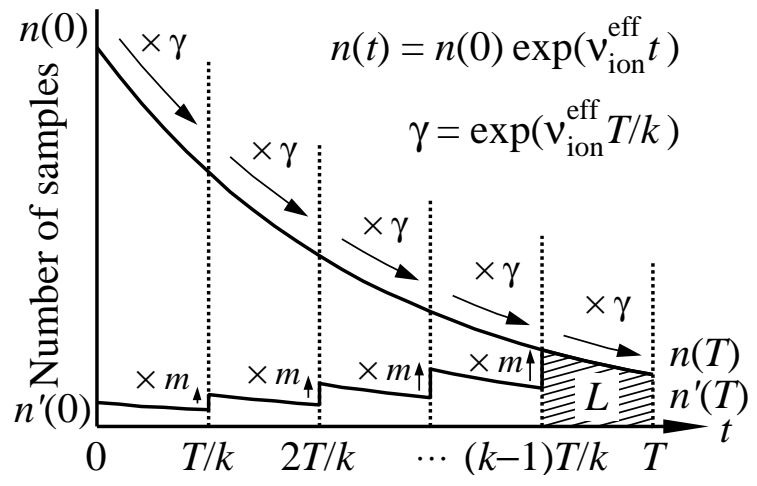

Fig. 1. Temporal variation of the number of samples under attachment-dominated condition. Exponential curve, $n(t)$ in conventional MCS; and stepwise curve, $n^{\prime}(t)$ in MCS with PSM $(m=2)$. The hatched area represents a unit computational load $L$ to quantify the load reduction efficiency.

and necessary to formulate its efficiency and limitation quantitatively for appropriate implementation.

Let us consider an attachment-dominated condition under which the number of electrons $n$ decreases exponentially with time $t$ as $n(t)=n(0) \exp \left(v_{\text {ion }}^{\text {eff }} t\right)$, where $v_{\text {ion }}^{\text {eff }}=v_{\text {ion }}-v_{\text {att }}<0$ is the effective ionization frequency, $v_{\text {ion }}$ and $v_{\text {att }}$ are the ionization and attachment frequencies, respectively. An electron swarm reaches its drift equilibrium at given $E, B$, and $N$ within a simulation time $T . n(T)$ is required to be statistically sufficient. Then, $n(0)=n(T) \exp \left(-v_{\text {ion }}^{\text {eff }} T\right)$ becomes correspondingly large if all electrons are tracked in a conventional manner. However, many of them vanish before the final sampling at $t=T$. The tracking of vanishing electrons is a waste of computational power, but we cannot determine which electrons would vanish prior to the choice by random numbers.

By PSM, one can produce $(m-1)$ copy samples that inherit the profile of an original sample every $T / k$, i.e., $(k-1)$ times at $t=i T / k(i=1,2, \ldots, k-1)$ (see Fig. 1). Here, the multiplication factor $m$ and the number of time divisions $k$ are natural numbers. At the time of copying, the weight of a sample is reduced to $1 / \mathrm{m}$ to maintain the continuity of the number of electrons. The copying adds no new statistical diversity at that time. However, the original and copy samples eventually blanch and undergo different histories after the succeeding collisions occur for $(m-1)$ of the $m$ original and copy samples. This blanching is substantial in PSM. Thus, $T / k$ should be sufficiently long to achieve the blanching. The blanching time $\tau_{\text {blanch }}$ is estimated from mean free time $\tau_{\text {free }}$ as $\tau_{\text {blanch }} \gg \tau_{\text {free }}=$ $1 / v_{\text {total }}$, where $v_{\text {total }}$ is the total collision frequency. Here, the relaxation of electron swarms, mainly by energy relaxation, ${ }^{3,5}$ generally needs a longer time than $\tau_{\text {blanch }}$.

There are two ways of practical PSM implementation. When the memory capacity for arrays is sufficiently large to store the position $(x, y, z)$ and velocity $\left(v_{x}, v_{y}, v_{z}\right)$ of all samples simultaneously (e.g., about $4.5 \mathrm{~GB}$ per $10^{8}$ samples for the six variables in double precision), tracking can proceed synchronously to the samples. The profiles of newborn samples are stored into vacant elements of the arrays. Management of the arrays, e.g., the search for vacancies and the judgement of occupation at sampling, is needed as an additional task. Otherwise, the memory can be saved by tracking samples 


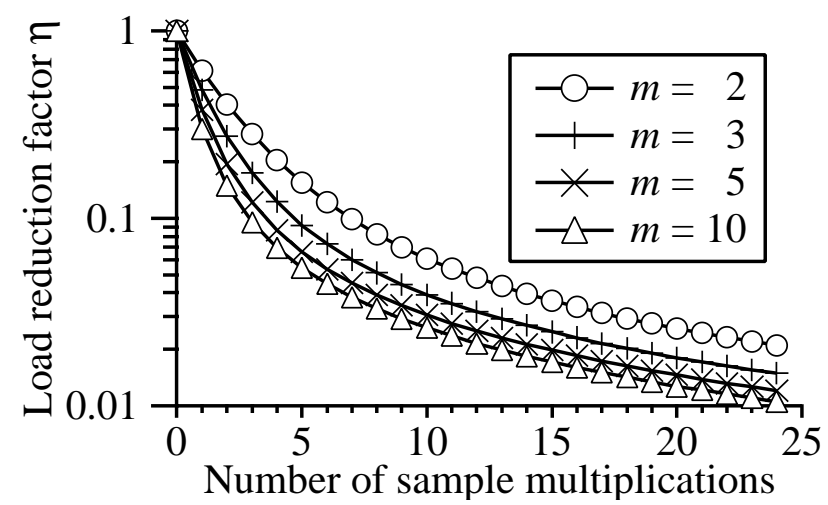

Fig. 2. Dependences of the load reduction factor $\eta$ on $m$ and $k . \eta$ was calculated at $v_{\text {ion }}^{\text {eff }}=-10^{7} \mathrm{~s}^{-1}$ and $T=250$ ns; i.e., $n(T) / n(0)=\exp (-2.5)$.

one by one from $t=0$ to $T$ repeatedly. The profiles of newborn samples are stacked and their tracking is reserved during the tracking of the original sample. The stacked profile of a copy sample and its birth time are recalled when the tracking of the copy sample resumes. The recalls are repeated until all stacked profiles are processed. These schemes are similar to the treatment of the secondary electrons generated by ionization except that the scattering operation for the secondary electrons are not applied to the PSM newborn samples. The stack size must be more than $(m-1)(k-1)$ for the PSM, and a margin for the secondary electrons $\left(\gg v_{\text {ion }} T\right)$ is necessary.

The computational load is proportional to the number of tracked samples. The load for conventional MCS $L_{\text {conv }}$ and that for PSM-MCS $L_{\mathrm{PSM}}$ are quantified with a unit load $L=\int_{[(k-1) / k] T}^{T} n(t) \mathrm{d} t$. From the relationships illustrated in Fig. 1, we obtain the loads and the load reduction factor $\eta$ as

$$
\begin{aligned}
\gamma & =\exp \left(v_{\mathrm{ion}}^{\mathrm{eff}} T / k\right)<1, \\
L_{\mathrm{conv}} & =\sum_{i=1}^{k}\left(\frac{1}{\gamma}\right)^{i-1} L=\frac{1-\gamma^{-k}}{1-\gamma^{-1}} L, \\
L_{\mathrm{PSM}} & =\sum_{i=1}^{k}\left(\frac{1}{m \gamma}\right)^{i-1} L=\frac{1-(m \gamma)^{-k}}{1-(m \gamma)^{-1}} L, \\
\eta & =\frac{L_{\mathrm{PSM}}}{L_{\mathrm{conv}}}=\frac{1-(m \gamma)^{-k}}{1-(m \gamma)^{-1}} \frac{1-\gamma^{-1}}{1-\gamma^{-k}} .
\end{aligned}
$$

Figure 2 shows $\eta$ plotted at various $m$ and $k$ values. Larger $m$ and $k$ are more desirable for load reduction, but the decrease in $\eta$ slows down with increasing $m$ and $k$.

There are restrictions on $m$ and $k$. One is that the number of collisionless electrons should be zero or one among $m$ original/copy electrons for the blanching. The probability $p$ that an electron remains collisionless for $T / k$ is $p=\exp \left(-v_{\text {total }} T / k\right)$. The requirement is satisfied when $P=(1-p)^{m}+m p(1-$ $p)^{m-1} \simeq 1$. If we choose, e.g., $P \geq 1-1 / 10000$ at $m=2$,

$$
k \leq 2 v_{\text {total }} T / \ln 10000 .
$$


Another is $n^{\prime}(0)=n^{\prime}(T) \exp \left(-v_{\text {ion }}^{\text {eff }} T\right) m^{-(k-1)}>1$ to avoid overpopulation, i.e., an unnecessarily large $n^{\prime}(T)$. This becomes

$$
k<1+\left[\ln n^{\prime}(T)-v_{\text {ion }}^{\mathrm{eff}} T\right] / \ln m .
$$

$T, v_{\text {total }}$, and $v_{\text {ion }}^{\text {eff }}$, which are necessary for the PSM setting, can be estimated from small-scale test MCSs.

A benchmark was performed for electron swarms in an electronegative gas, $\mathrm{SF}_{6}$, in $\mathbf{E} \perp \mathbf{B}$ at a low $E / N$ of $100 \mathrm{Td}$ (townsend, $1 \mathrm{Td}=10^{-21} \mathrm{Vm}^{2}$ ) and a high $B / N$ of $1000 \mathrm{Hx}$ (huxley, $1 \mathrm{Hx}=10^{-27} \mathrm{Tm}^{3}$ ), at $N=10^{22} \mathrm{~m}^{-3}$. This condition is strongly attachment-dominated. The initial electrons were released with a Maxwellian EVDF with a mean energy of $1 \mathrm{eV}$. The electron trajectories were calculated on the basis of Ref. 6. A $\Delta t$ method with $\Delta t=0.1 \mathrm{ps}$ and a timesaving scheme ${ }^{7}$ were adopted for the judgement of the occurrence of collisional events. The collision cross sections of $\mathrm{SF}_{6}$ were taken from Refs. 8 and 9. The workstation used for the benchmark had the following properties: CPU, Intel Xeon E5-1650 v4, 3.60 GHz; main memory, $128 \mathrm{~GB}$; operating system, Linux CentOS 7.3; and programming language, $\mathrm{C}++$.

Figure 3 shows the relaxation of representative electron transport coefficients obtained by the conventional MCS with $n(T) \geq 10^{6}$; the mean electron energy $\langle\varepsilon\rangle=\frac{1}{2} m_{\mathrm{e}}\left\langle v_{x}^{2}+v_{y}^{2}+v_{z}^{2}\right\rangle$, the average velocity $V=\left\langle v_{z}\right\rangle$, the centroid drift velocity $W=d\langle z\rangle / d t$, and the diffusion coefficient $D=\frac{1}{2} d\langle(z-$ $\left.\langle z\rangle)^{2}\right\rangle / d t$. Here, $m_{\mathrm{e}}$ is the electronic mass, $\langle X\rangle$ represents the ensemble average of a quantity $X$ among the electrons in the swarm, and $-\mathbf{E}$ is applied to the $+z$-direction. The quantities were calculated in double precision. The fluctuation was large for the $z$-dependent coefficients $W$ and $D$. Their standard deviations during the final $T / 10$ period, before which the swarm seems to have reached its equilibrium, are listed in Table I as relative values $\sigma_{X} / X$ together with other results.

Saving time and suppression of the statistical fluctuation were attempted by PSM at $m=2,3$, and 5. Cases $n^{\prime}(T) \geq 10^{6}$ and $n^{\prime}(T) \geq 10^{7}$ were examined for comparison of $\sigma_{X} / X$. $v_{\text {total }}$ was about $2.2 \times 10^{9} \mathrm{~s}^{-1}$, thus, $\tau_{\text {free }}=0.45 \mathrm{~ns}$, and $\nu_{\text {ion }}^{\text {eff }}=-1.0 \times 10^{7} \mathrm{~s}^{-1} \cdot k$ was chosen as listed in Table I. $k=25$ is close to the upper limit of $k$ in Eq. (6) for $n^{\prime}(T) \geq 10^{7}$, which is tighter than Eq. (5). The initial $n(0)$ and $n^{\prime}(0)$, the final $n(T)$ and $n^{\prime}(T)$, and the CPU time $T_{\mathrm{CPU}}$ spent for each MCS are shown together. The equilibrium values of $X$ and $\sigma_{X} / X$ in cases 2-9 were calculated in the final $T / k$ period. They agreed with each other satisfactorily, and the EVDFs did as well.

Let us focus on the fluctuation in $D$ because $\sigma_{D} / D$ was the largest among the coefficients in Fig. 3. Figure 4 shows fluctuations of $N D$ during the relaxation process in the simplest PSM at $m=2$. The early fluctuation in case 1 is the smallest for the largest $n$. In Table I, $\sigma_{D} / D$ in the cases of $n^{\prime}(T) \geq 10^{6}$ was comparable to that in case 1 , but $T_{\mathrm{CPU}}$ was shortened much by PSM. The time saved can be spent to increase $n^{\prime}(T)$ for a smaller fluctuation in the final period. The $T_{\mathrm{CPU}}$ values in the cases of $n^{\prime}(T) \geq 10^{7}$ were still shorter than that in case 1 . Although the initial samples in cases 4 and 5 were less diverse than those in the other cases and their early relaxation processes were no longer clearly observable, $N D$ reached appropriate equilibrium values by the final $T / k$. Overpopulation in $n^{\prime}(T)$ was 


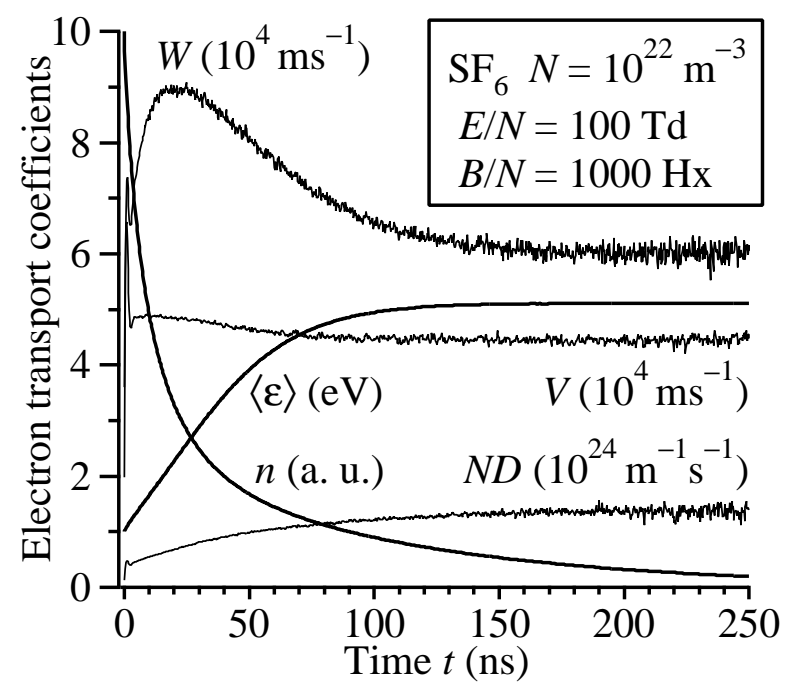

Fig. 3. Relaxation of electron transport coefficients observed by the conventional MCS with $n(T) \geq 10^{6}$. $\langle\varepsilon\rangle=5.114 \mathrm{eV}, V=4.45 \times 10^{4} \mathrm{~ms}^{-1}, W=6.02 \times 10^{4} \mathrm{~ms}^{-1}$, and $N D=1.37 \times 10^{24} \mathrm{~m}^{-1} \mathrm{~s}^{-1}$.

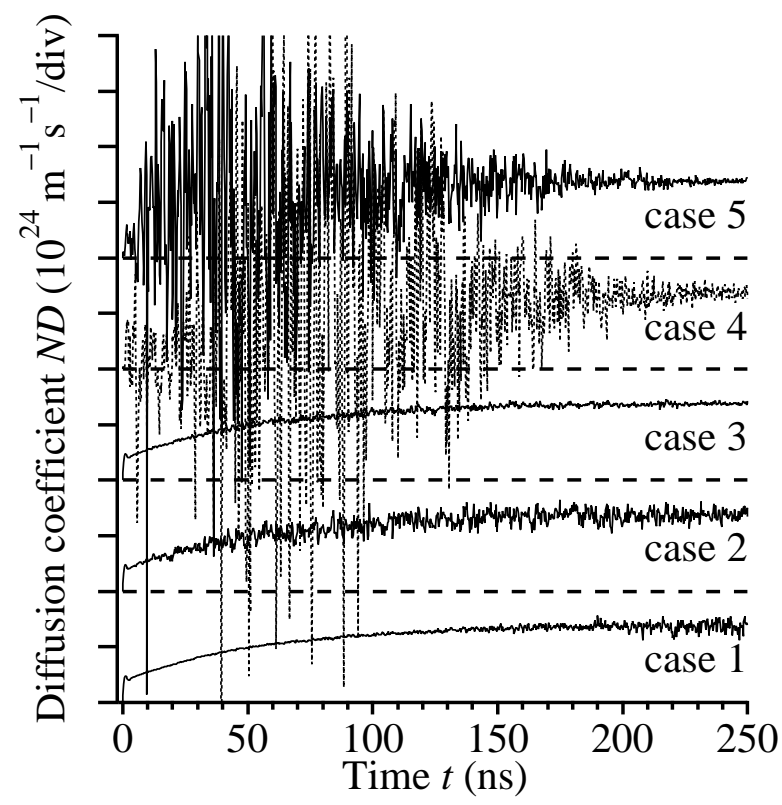

Fig. 4. Comparison of statistical fluctuations in the relaxation of $N D$. The curve of case 1 is identical to that in Fig. 3. Cases 2-5 were shifted upward in steps of two divisions to show them separately. Case 4 is plotted as a broken curve to distinguish it from the other crossing curves.

observed in cases with small $n^{\prime}(0)$, and $T_{\mathrm{CPU}}$ values ranged wider in such cases.

By PSM, the histories of individual samples that have survived a relaxation period under an attachment-dominated condition are copied before they are lost uselessly. This enhances the opportunity that each history participates in the final statistics. The samples produced by PSM are the descendants of a limited number of initial samples. Therefore, one may be concerned about some bias by 
analogy with the statistical time lag in the early phase in electric breakdown that an electron avalanche develops from a few seed electrons under an ionization-dominated condition. However, it seems that a satisfactory randomization has been achieved during sufficiently long intervals of PSM for the blanching of copy samples. As long as PSM is applied equally to all of the samples surviving at that time, bias due to factitious choices is excluded from the final statistics.

The efficiency and limitation of the PSM were discussed and quantitative implementation guidelines were presented. It was demonstrated that PSM enables us to reduce the computational load in MCSs even under a severe attachment-dominated condition and the statistical fluctuation can be reduced substantially by utilizing the saved CPU time for increasing the number of samples.

\section{Acknowledgment}

This work was supported by JSPS KAKENHI Grant Number JP16K05626. 


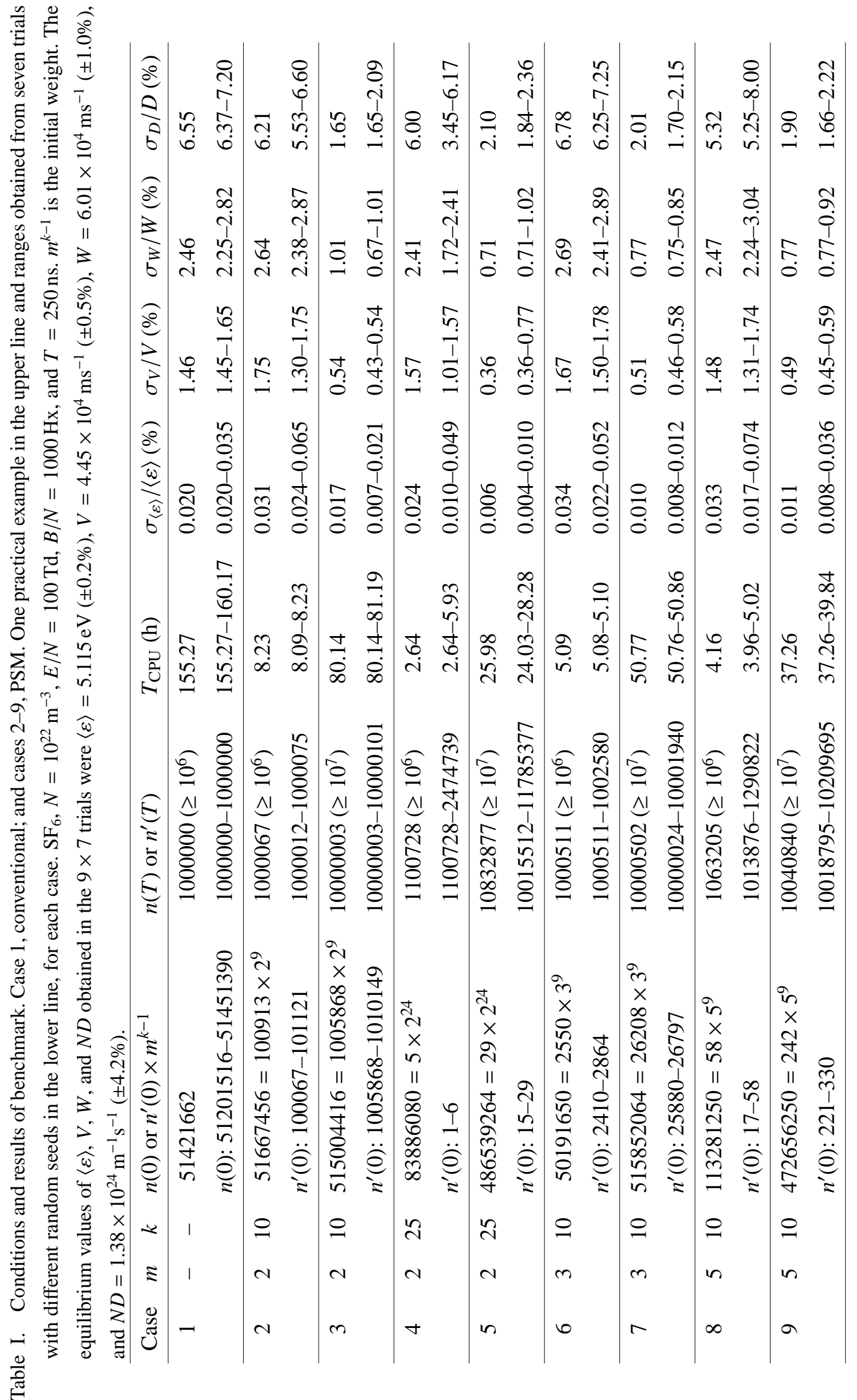




\section{References}

1) R. D. White, M. J. Brennan, and K. Ness, J. Phys. D 30, 810 (1997).

2) H. Sugawara and Y. Sakai, Japan. J. Appl. Phys. 45, 5189 (2006).

3) H. Sugawara, Plasma Sources Sci. Technol. 26, 044002 (2017).

4) J. Mirić, D. Bošnjaković, I. Simonović, Z. Lj. Petrović, and S. Dujko, Plasma Sources Sci. Technol. 25, 065010 (2016).

5) T. Makabe, N. Nakano, and Y. Yamaguchi, Phys. Rev. A 45, 2520 (1992).

6) H. Sugawara, T. Yahata, A. Oda, and Y. Sakai, J. Phys. D 33, 1191 (2000).

7) H. Sugawara, N. Mori, Y. Sakai, and Y. Suda, J. Comput. Phys. 223, 298 (2007).

8) H. Itoh, Y. Miura, N. Ikuta, Y. Nakao, and H. Tagashira, J. Phys. D 21, 922 (1988).

9) H. Itoh, T. Matsumura, K. Satoh, H. Date, Y. Nakao, and H. Tagashira, J. Phys. D 26, 1975 (1993). 